\title{
Mice lacking $\beta 3$ integrins are osteosclerotic because of dysfunctional osteoclasts
}

\author{
Kevin P. McHugh, ${ }^{1}$ Kairbaan Hodivala-Dilke, ${ }^{2}$ Ming-Hao Zheng, ${ }^{1,3}$ \\ Noriyuki Namba, ${ }^{1}$ Jonathan Lam, ${ }^{1}$ Deborah Novack, ${ }^{1}$ Xu Feng, ${ }^{1}$ F. Patrick Ross, ${ }^{1}$ \\ Richard O. Hynes, ${ }^{2}$ and Steven L. Teitelbaum ${ }^{1}$ \\ ${ }^{1}$ Department of Pathology, Washington University School of Medicine, St. Louis, Missouri 63110, USA
${ }^{2}$ Howard Hughes Medical Institute, Center for Cancer Research and Department of Biology, Massachusetts Institute of
Technology, Cambridge, Massachusetts 02139, USA
${ }^{3}$ Department of Orthopedic Surgery, University of Western Australia, Nedlands, Western Australia 6009, Australia
}

Address correspondence to: Steven L. Teitelbaum, Washington University School of Medicine, Barnes-Jewish Hospital North, 216 South Kingshighway, Mail Stop 9031649, St. Louis, Missouri 63110, USA. Phone: (314) 454-8463; Fax: (314) 454-5505;

E-mail: teitelbs@medicine.wustl.edu.

Received for publication November 11, 1999, and accepted in revised form January 10, 2000.

\begin{abstract}
Osteoclasts express the $\alpha v \beta 3$ integrin, an adhesion receptor that has been implicated in bone resorption and that is therefore a potential therapeutic target. To assess the role of this heterodimer in skeletal development in vivo, we engineered mice in which the gene for the $\beta 3$ integrin subunit was deleted. Bone marrow macrophages derived from these mutants differentiate in vitro into numerous osteoclasts, thus establishing that $\alpha v \beta 3$ is not necessary for osteoclast recruitment. Furthermore, the closely related integrin, $\alpha v \beta 5$, does not substitute for $\alpha v \beta 3$ during cytokine stimulation or authentic osteoclastogenesis. $\beta 3$ knockout mice, but not their heterozygous littermates, develop histologically and radiographically evident osteosclerosis with age. Despite their increased bone mass, $\beta 3$-null mice contain 3.5-fold more osteoclasts than do heterozygotes. These mutant osteoclasts are, however, dysfunctional, as evidenced by their reduced ability to resorb whale dentin in vitro and the significant hypocalcemia seen in the knockout mice. The resorptive defect in $\beta 3$ deficient osteoclasts may reflect absence of matrix-derived intracellular signals, since their cytoskeleton is distinctly abnormal and they fail to spread in vitro, to form actin rings ex vivo, or to form normal ruffled membranes in vivo. Thus, although it is not required for osteoclastogenesis, the integrin $\alpha v \beta 3$ is essential for normal osteoclast function.
\end{abstract}

J. Clin. Invest. 105:433-440 (2000).

\section{Introduction}

The osteoclast, a polykaryon of monocyte/macrophage origin, is probably the exclusive resorptive cell of bone. As any type of osteoporosis always reflects enhanced resorption relative to formation, the osteoclast has been a principal therapeutic target in circumstances of diminished bone mass. In fact, all successful antiosteoporosis agents identified to date target the osteoclast. Despite success achieved with antiresorptive drugs such as estrogen and newer compounds like bisphosonates, each carries, unfortunately, the potential for substantial complications. However, recent years have witnessed identification of novel, osteoclast-expressed molecules that may serve as potential therapeutic targets. The success of this search is reflective of the insights gained into osteoclast formation mechanisms and the means by which the cell resorbs bone. It is now known that osteoclasts differentiate from monocyte/macrophage precursors under the influence of 2 essential molecules, namely the receptor activator of NF- $\mathrm{KB}$ ligand (RANKL) (equivalent to osteoprotegerin ligand) and the macrophage colony stimulating factor (M-CSF) (1). When the differenti- ated polykaryon contacts bone, it undergoes cytoskeletal reorganization eventuating in polarization of its resorptive apparatus to the cell-bone interface creating, thereof, an isolated, resorptive microenvironment (2). Thus, matrix-derived intracellular signals and physical intimacy of the osteoclast with underlying bone, are central to the cell's capacity to degrade the skeleton. Hence, osteoclast-residing, matrix attachment molecules - particularly those capable of transmitting matrix-derived signals - might serve as potential antiosteoporosis targets.

Based on blocking experiments, the $\alpha v \beta 3$ integrin has been identified as a major functional adhesion receptor on osteoclasts. Specifically, inhibitors of $\alpha v \beta 3$, particularly peptides or peptidomimetics, reduce the capacity of osteoclasts to bind to and resorb bone $(3,4)$. These observations suggest that $\alpha v \beta 3$ plays a major role in osteoclast function $(3,4)$ and generation (5), and that inhibitors of this integrin may be useful in preventing osteoporosis (4).

By application of similar inhibitors, $\alpha v \beta 3$ has also been implicated in angiogenesis (6). Genetic ablation of either $\alpha v(7)$ or $\beta 3$ (8) fails, however, to block angiogenesis. 
Thus, despite observations made with $\alpha v \beta 3$ inhibitors, these studies not only establish that $\alpha v \beta 3$ is not essential for angiogenesis, but also raise questions regarding the validity of the osteoclast-based blocking experiments. This conundrum, taken with the possibility that $\alpha v \beta 3$ may indirectly regulate the functions of other integrins by so-called "transdominant regulation" (9), indicates that further exploration of the role of $\alpha v \beta 3$ in osteoclastic bone resorption is essential. To that end, we generated $\beta 3^{-/-}$mice using a targeting construct in which a 1.2-kb genomic fragment (including $300 \mathrm{bp}$ of the promoter, the transcriptional start site, exon I, intron I, and exon II) is replaced by a phosphoglycerokinase promoter-neomycin resistance gene cassette (8). $\beta 3$-null mice are viable and exhibit the bleeding disorders expected as a consequence of their lack of $\alpha \operatorname{Irb} \beta 3$ integrin on platelets (8). Here we report our analyses of bone structure and metabolism in these mice. Our findings demonstrate that although $\alpha v \beta 3$ crucially - but incompletely - regulates osteoclast function (which is in contrast to results obtained with inhibitors of the integrin [5]), it is not necessary for osteoclast generation.

\section{Methods}

Materials. Recombinant murine IL-4 and GM-CSF were purchased from Genzyme Pharmaceuticals (Cambridge, Massachusetts, USA). 1,25-Dihyroxyvitamin $\mathrm{D}_{3}$ was a gift of Milan Uscosovich (Hoffman LaRoche Inc., Nutley, New Jersey, USA). Murine M-CSF was generated and purified in our laboratory as described previously (10). Glutathione-S-transferase (GST)-RANKL

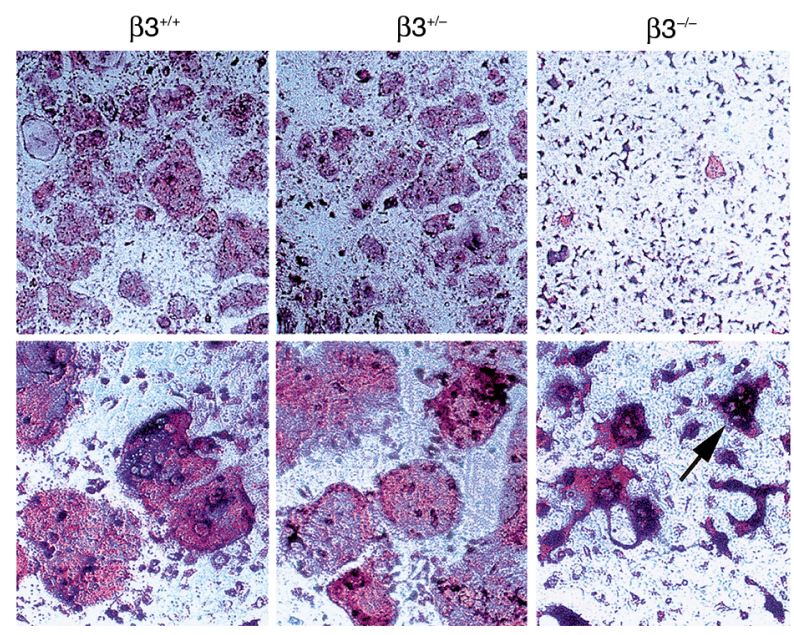

\section{Figure 1}

$\beta 3^{-/-}$BMMs differentiate into osteoclasts that fail to spread. Sevenday osteoclastogenic cultures, containing BMMs of 2-month-old female littermates and ST2 stromal cells, were stained for TRAP activity (red reaction product). $\beta 3^{+/+}$and $\beta 3^{+/-}$BMMs form numerous well spread, TRAP-expressing giant cells. TRAP-expressing cells in cultures with $\beta 3^{-/-}$BMMS, in contrast, fail to spread $(\times 100)$. High magnification of the same cultures (bottom row) demonstrates that the TRAP-expressing giant cells, in each circumstance, contain multiple nuclei (arrow) $(\times 250)$. was expressed in our laboratory. The GST-RANKL vector was constructed by PCR cloning a SalI/NotI fragment corresponding to amino acids 158-316 of murine RANKL from osteoblast cDNA (forward primer: 5'-ATGTGGCCCGTCGACGCAAGCCTGAGGCCCAGCC-3'; reverse primer: 5'-AAACATCTAGGGCGGCCGCGCTAATG- $3^{\prime}$ ) and cloning into pGEX-4T-1 (Amersham Pharmacia Biotech, Piscataway, New Jersey, USA). Both strands were sequenced. High-expressing BL21 (Stratagene, La Jolla, California, USA) cells were lysed under nondenaturing conditions and GSTRANKL was purified over a glutathione-Sepharose column followed by ion exchange chromatography.

Osteoclast generation. Bone marrow macrophages (BMMs) were isolated from whole marrow of 4- to 8week-old mice, cultured overnight, and then subjected to Ficoll-Hypaque gradient purification as described (11). Cells at the gradient interface were collected and cultured on tissue culture plates or dentin slices at a 10:1 ratio with the ST2 stromal cell line in $\alpha$ minimal essential medium containing 1,25-dihydroxyvitamin $\mathrm{D}_{3}$ and glucocorticoids, supplemented with $10 \%$ heatinactivated fetal bovine serum at $37^{\circ} \mathrm{C}$ with $5 \% \mathrm{CO}_{2}$ (12). In some experiments, pure populations of BMMs (13) were maintained with RANKL $(100 \mathrm{ng} / \mathrm{mL})$ and M-CSF $(10 \mathrm{ng} / \mathrm{mL})$, which induces these cells to undergo osteoclast differentiation (1).

Histology. Mouse femurs were excised, cleaned of soft tissue, and decalcified in EDTA. Histologic sections were stained with hematoxylin and eosin or for tartrate-resistant acid phosphatase (TRAP) activity using a commercial kit (Sigma Chemical Co., St. Louis, Missouri, USA). Generated osteoclasts were stained for TRAP activity using the same kit. The number of osteoclasts per millimeter of trabecular bone surface was determined using the Bioquant system (R\&M Biometrics, Nashville, Tennessee, USA).

Cell surface protein biotinylation. For 5 days, $10^{7} \mathrm{BMMs}$ were grown in $\alpha$-MEM $+10 \%$ FCS containing 1,000 $\mathrm{U} / \mathrm{mL} \mathrm{M-CSF}$ (stage-1) and then treated with IL-4 (5 $\mathrm{ng} / \mathrm{mL}$ ) (R \& D Systems Inc., Minneapolis, Minnesota, USA) or vehicle for an additional 48 hours. Cells were washed in PBS-0.1M HEPES, and cell surface proteins were biotinylated using n-hydroxysuccinimide-biotin (NHS-biotin, Pierce Chemical Co., Rockford, Illinois, USA) and the manufacturer's recommended conditions $(450 \mu \mathrm{g}$ NHS-biotin in $2.5 \mathrm{~mL}$ PBS-0.1 M HEPES per $150 \mathrm{~mm}^{2}$ plate, and then incubated for 45 minutes at room temperature). Cells were washed gently 3 times with cold PBS and then lysed in lysis buffer (10 $\mathrm{mM}$ Tris, $150 \mathrm{mM} \mathrm{NaCl}, 1.0 \mathrm{mM} \mathrm{CaCl}, 0.02 \% \mathrm{NaN}_{3} 2 \times$ protease inhibitor cocktail, and 2\% Renex (Sigma Chemical Co.). Protein concentrations in the lysates were determined by bicinchoninic acid reaction (Pierce Chemical Co.), and equal amounts of total protein were immunoprecipitated.

Immunoprecipitation. Immunoprecipitation was carried out essentially as previously described (10). Lysates were precleared for 1 hour by incubation at 


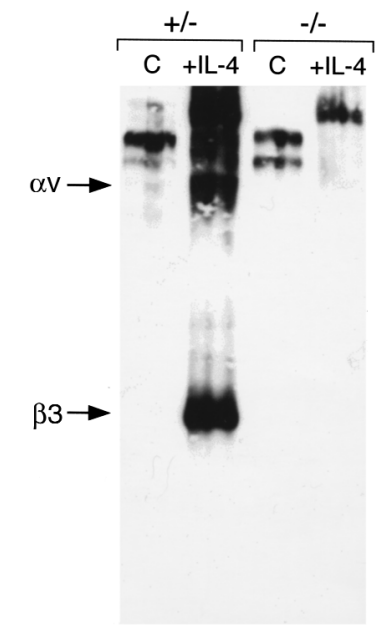

Figure 2

$\beta 3^{-1-}$ BMMs fail to express $\alpha v \beta 3$ and the integrin is not induced by IL-4. Pure populations of $\beta 3^{+/-}$or $\beta 3^{-/-}$BMMs were treated for 48 hours with vehicle $(C)$ or murine IL-4 in an amount $(5 \mathrm{ng} / \mathrm{mL})$ known to optimally enhance $\beta 3$ expression (10). The cells were then surface labeled with NHS-biotin and lysed, and then the lysate was immunoprecipitated with hamster antimurine $\beta 3 \mathrm{mAb}$ and the immunoprecipitate subjected to SDS-PAGE. Similar to wild-type BMMs (10), $\beta 3^{+/-}$cells express minimal $\alpha v \beta 3$ and the integrin is upregulated by IL-4. In contrast, $\beta 3^{-/-}$BMMs fail to express $\alpha v \beta 3$ in the presence or absence of IL-4.

$4^{\circ} \mathrm{C}$ with $100 \mu \mathrm{L} \gamma$-bind (Pharmacia Biotech $\mathrm{AB}$, Uppsala, Sweden) in lysis buffer. After centrifugation, supernatants were collected and precleared with nonspecific $\mathrm{Ab}$ by the addition of $5 \mu \mathrm{g}(5 \mu \mathrm{L})$ of hamster IgG (PharMingen, San Diego, California, USA) with $100 \mu \mathrm{L} \gamma$-bind and incubated for 1 hour at $4^{\circ} \mathrm{C}$. After centrifugation, pellets were washed 4 times in $0.5 \mathrm{~mL}$ lysis buffer, and then finally resuspended in $75 \mu \mathrm{L}$ gel loading buffer and boiled for 5 minutes before electrophoresis.

Blotting and detection. Western blots were carried out as described previously (11). Precipitated proteins were resolved by $8 \%$ SDS-PAGE. Proteins were electroblotted on nitrocellulose, and membranes were blocked in 10\% skim milk, prepared in PBS with $0.05 \%$ Tween-20. Biotinylated proteins were detected by the addition of avidin-horseradish peroxidase conjugate (Pierce Chemical Co.), to $2 \mu \mathrm{g} / \mathrm{mL}$ in $10 \%$ milk, PBS, and $0.05 \%$ Tween-20 for 1 hour at room temperature. Membranes were washed extensively (5 times for 15 minutes) in PBS- $0.05 \%$ Tween, and the blot was developed for horseradish peroxidase activity by enhanced chemiluminescence (ECL) (Amersham Life Sciences Inc., Arlington Heights, Illinois, USA).

Northern analysis. After Ficoll-Hypaque gradient purification, BMMs were cultured for 4 days in the presence of GM-CSF $(10 \mathrm{ng} / \mathrm{mL})$, and an optimal amount of M-CSF (purified from L929 conditioned media) and recombinant GST-osteoprotegerin ligand (OPGL) $(2 \mathrm{ng} / \mathrm{mL})$ generated in our laboratory. Total
RNA was isolated using Trizol (GIBCO BRL, Gaithersburg, Maryland, USA), and then analyzed using NorthernMax (Ambion Inc., Austin, Texas, USA). Nonisotopic probes were generated using the BrightStar kit (Ambion Inc.); GAPDH control probe was supplied by the manufacturer, murine $\beta 3$ integrin probe consisted of a 537-nucleotide BamHI/HindIII fragment at the $5^{\prime}$ end of the cDNA (14), murine $\beta 5$ integrin probe consisted of a 1-kb Sall fragment of cDNA (15).

Radiography. Four-month-old $\beta 3^{-/-}$mice were simultaneously radiographed with a $\beta 3^{+/+}$or $\beta 3^{+/-}$littermate using mammography film.

Transmission electron microscopy. Long bones from 4- to 5-day-old $\beta 3^{+/-}$and $\beta 3^{-/-}$mice were dissected free of soft tissue and fixed with $2.5 \%$ glutaraldehyde and $4 \%$ paraformaldehyde in $0.01 \mathrm{M}$ cacodylate buffer for at least 10 minutes. They were then washed and postfixed with $1 \% \mathrm{OsO}_{4}$, dehydrated, and then embedded in araldite (Polyscience, Worhtington, Pennsylvania, USA). Sections were cut on an ultramicrotome and examined on an electron microscope (410; Philips Electron Optics, Mahwah, New Jersey, USA).

Examination of actin cytoskeleton. Long bones from 4to 5-day-old mice were dissected free of soft tissue and sectioned longitudinally. They were placed in PBS in pyrocarbonate (DEPC)-treated water. Adherent marrow cells were removed with a fine paint brush. The bones were imprinted with minimal pressure onto salinated glass slides. The slides were air-dried at room temperature for 15 minutes, fixed with 3.7\% formaldehyde, and then stained for TRAP by incubating with Naphthol AS-BI phosphate in the presence of $100 \mathrm{nM}$ of sodium tartrate (osteoclasts were identified by expression of TRAP). To examine the Factin distribution within osteoclasts, slides were treated with $0.1 \%$ Triton X-100 in PBS for 5 minutes and reacted with rhodamine phalloidin. A confocal microscope (MRC-1000; Bio-Rad Laboratories Inc., Hercules, California, USA) equipped with a PlanApo $60 \times$ 1.4 NA objective was used to assess the cytoplasmic

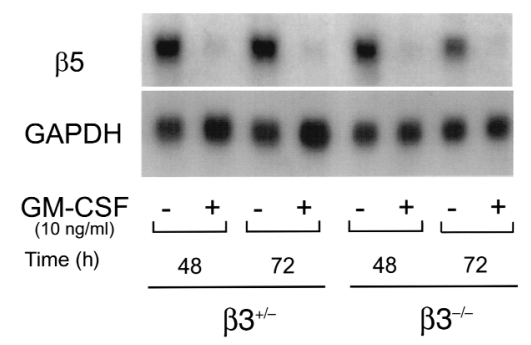

\section{Figure 3}

The $\beta 5$ integrin subunit does not compensate for absence of $\beta 3$ in GM-CSF-treated $\beta 3^{-/-}$BMMs. Pure populations of $\beta 3^{+/-}$or $\beta 3^{-/-}$BMMs were treated for 48 or 72 hours with vehicle (-) or murine GM-CSF $(+)$ in an amount $(10 \mathrm{ng} / \mathrm{mL})$ known to optimally suppress $\beta 5$ mRNA expression by wild-type BMMs (17). Total RNA was isolated and subjected to Northern analysis using a murine $\beta 5$ cDNA. The cytokine inhibits $\beta 5$ mRNA expression equally in $\beta 3^{+/-}$and $\beta 3^{-/-}$BMMs. 


\section{Figure 4}

Compensatory upregulation of $\beta 5$ integrin does not occur in $\beta 3^{-/-}$ osteoclasts. Wild-type (WT) and $\beta 3^{-/-}$BMMs were grown in the presence of M-CSF alone or in combination with OPGL for 4 days. Ten micrograms of total RNA was run in each lane for Northern analysis. The blot was probed first with a combination of $\beta 5$ and GAPDH probes, then stripped and probed with $\beta 3$. In WT cultures, OPGL upregulates expression of $\beta 3$ and downregulates expression of $\beta 5$. In $\beta 3^{-1-}$ cultures, no $\beta 3$ mRNA is seen, and the downregulation of $\beta 5$ is equivalent to that seen in WT cultures.

distribution of F-actin in TRAP-positive cells. The wavelength for excitation and emission for F-actin was $554 \mathrm{~nm}$ and $573 \mathrm{~nm}$, respectively.

Bone resorption. Osteoclasts were generated in $\mathrm{BMM} / \mathrm{ST} 2$ coculture on dentin slices for 7-10 days. Cells were removed from the dentin slices with $0.25 \mathrm{M}$ ammonium hydroxide and mechanical agitation. Dentin slices were then subjected to scanning electron microscopy (16). Maximum resorption lacuna depth was measured using a confocal microscope (Microradiance; Bio-Rad Laboratories Inc.). Images were collected in 514-nm argon laser light with epireflection.

\section{Results}

$\beta 3^{--}$BMMs differentiate into osteoclasts that fail to spread. Similar to murine BMMs expressing the integrin subunit, osteoclasts can be generated from those that are $\beta 3$ null (Figure 1). Within 7 days of coculture with the marrow stromal line, ST2, in the presence of 1,25-dihydroxyvitamin $\mathrm{D}_{3}$ and glucocorticoids, both wild-type and heterozygous BMMs differentiate into well spread, TRAP-expressing polykaryons (in all circumstances, $\mathrm{B3}^{+/+}$and $\mathrm{B3}^{+/-}$BMMs yield similar results; thus, heterozygous or wild-type cells were utilized in various experiments on the basis of litter availability). $\beta 3$-null macrophages also develop into numerous TRAP-positive multinucleated cells, but these fail to spread properly. $\beta 3^{-1-}$ BMMs fail to express $\alpha v \beta 3$, and the integrin is not induced by $I L-4$. $\alpha v \beta 3$ is minimally expressed by BMMs and appears as the cells assume the osteoclast phenotype (17) or are exposed to cytokines such as IL-4, which induces the integrin by transactivation of the $\beta 3$ gene (10). To confirm that mutant BMMs lack the capacity to express $\alpha v \beta 3$, we exposed $\beta 3^{+/-}$and $\beta 3^{-/-}$ cells to IL-4 (50 units/mL) for 36 hours (10). The cells were surfaced labeled and lysed. The lysate was immunoprecipitated with a $\beta 3$-specific antibody, and the immunoprecipitate was analyzed by SDS-PAGE. As expected, untreated heterozygous and mutant BMMs fail to express $\alpha v \beta 3$ (Figure 2). Whereas the integrin is abundantly induced in cytokine-exposed heterozygous cells, $\alpha v \beta 3$ remains undetectable in $\beta 3$ knockout BMMs even in the presence of IL-4.

The $\beta 5$ integrin subunit does not compensate for absence of $\beta 3$. Although they do not express $\alpha v \beta 3$, immature osteoclast precursors express $\alpha_{\mathrm{v}} \beta 5$, which, like $\alpha \mathrm{v} \beta 3$,

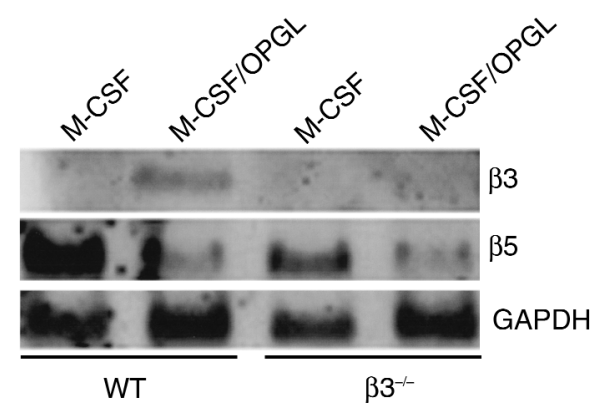

recognizes the RGD amino acid motif (17). We have shown that as BMMs differentiate into mature osteoclasts in vitro, $\alpha v \beta 5$ is replaced by $\alpha v \beta 3$ (17). This observation raised the possibility that the absence of $\beta 3$ integrin, in differentiating osteoclast precursors, might be compensated by persistence of $\alpha v \beta 5$. To determine if this is the case, we took advantage of the capacity of GM-CSF to reciprocally modulate $\beta 5$ and $\beta 3$ integrin expression, by BMMs, in a manner mirroring osteoclastogenesis (17). In fact, GM-CSF prompts disappearance of $\beta 5$ mRNA in mutant as well as heterozygous BMMs (Figure 3 ). Thus, $\alpha v \beta 5$ does not substitute for $\alpha v \beta 3$ when the latter is absent in cytokine-stimulated osteoclast precursors.

We next asked if $\beta 3$ mRNA is absent and the $\beta 5$ message is enhanced in $\beta 3$ knockout cells, in the context of authentic osteoclastogenesis. To this end, we used RANKL which, in the presence of M-CSF, prompts BMM differentiation into osteoclasts (1). Thus, $\beta 3^{+/+}$ and $\beta 3^{-/-}$BMMs were treated with M-CSF alone or MCSF plus RANKL. After 4 days, RNA was probed by Northern blot analysis using murine $\beta 3$ and $\beta 5$ cDNAs. $\beta 5$ mRNA is abundant in wild-type and mutant cells treated only with M-CSF and diminishes, in both strains, with addition of RANKL (Figure 4). As expected, $\beta 3$ message is enhanced by RANKL in wild-type BMMs and fails to appear in similarly treated null cells. Thus, at least in vitro, $\alpha v \beta 5$ does not compensate for lack of $\alpha v \beta 3$ in $\beta 3^{-/-}$cells committed to osteoclast differentiation.

$\beta 3^{-1-}$ mice become osteosclerotic with age. The external appearance of $\beta 3$-null and wild-type mice, at birth and throughout life, is similar. On the other hand, as $\beta 3^{-/-}$ mice age, they become osteosclerotic. This increase in bone mass is radiographically evident by 4 months. At 6 months, radiographs of mutant tails invariably demonstrate increased bone density, and, by this means, we can reliably discriminate $\beta 3$-null from wild-type and heterozygous mice, the latter being indistinguishable from the wild-type in all parameters examined (Figure 5).

Confirming our radiographic observations, histologic sections of $\beta^{-/-}$bones reveal a markedly increased cortical and trabecular mass (Figure 6). On the other hand, the histologic hallmark of osteopetrosis, namely the persistence of cartilaginous bars, reflecting failure to resorb primary spongiosa (18), is 


\section{Figure 5}

$\beta 3^{-/-}$mice develop radiographic osteosclerosis. Tails of 36 month-old $\beta 3^{-/-}$mice and their heterozygous or wild-type sexmatched littermates were radiographed simultaneously to avoid technical artifacts. The mutant, in all circumstances, shows enhanced bone density.

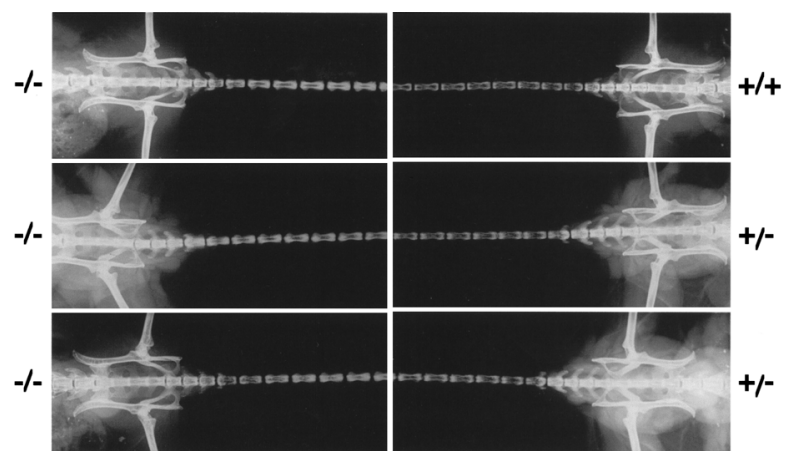

clasts is organized in circular structures consisting of podosomes or actin rings, in every mutant polykaryon examined, actin rings fail to form and actin is diffusely distributed, in a punctate pattern, throughout the cytoplasm (Figure 9). Therefore, $\alpha v \beta 3$ transmits matrix-derived signals that organize the osteoclast cytoskeleton.

Finally, we turned to the nature of the resorptive defect observed in $\beta 3$ knockout osteoclasts. To this end, we generated $\beta 3^{+/-}$and $\beta 3^{-/-}$osteoclasts from BMMs on whale dentin, and imaged the resulting lacunae by scanning electron microscopy. Heterozygous osteoclasts excavate numerous characteristic, well delineated resorptive "pits" on the dentin surface, whereas those produced by cells lacking $\alpha v \beta 3$ are shallow and poorly defined (Figure 10). Specifi-

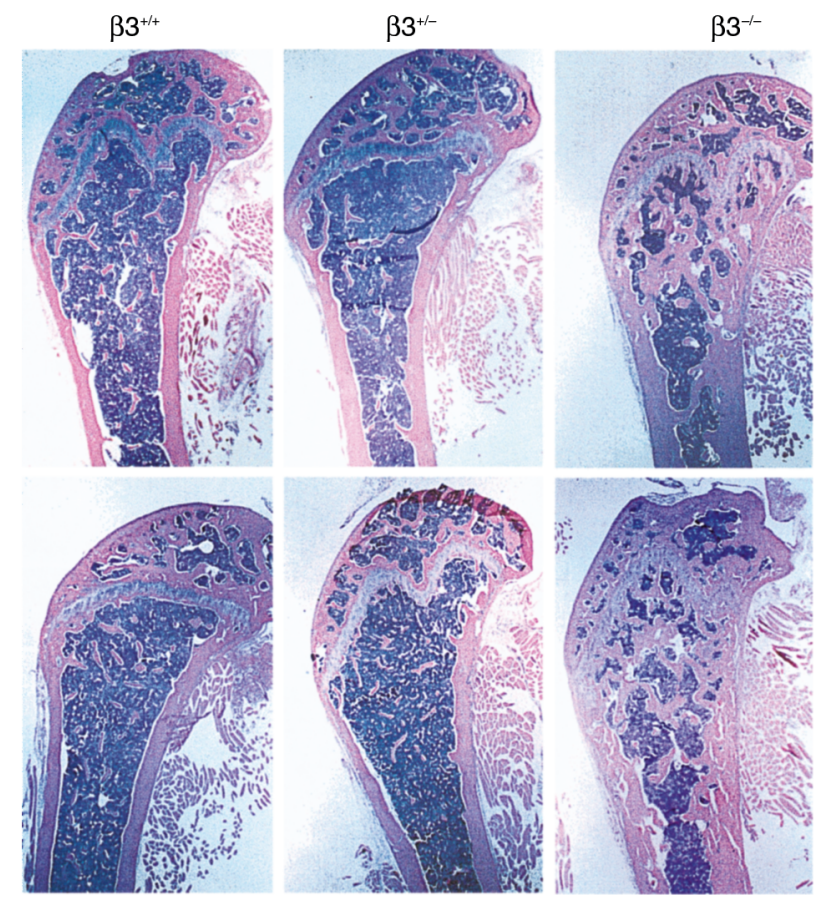

\section{Figure 6}

$\beta 3^{-/-}$mice develop histologic osteosclerosis. Distal femora of 2 sets of 6-month-old, sex-matched, $\beta 3^{+/+}, \beta 3^{+/}$, and $\beta 3^{-/-}$littermates. Note the marked increase in cortical and trabecular bone mass in $\beta 3^{-/-}$mice as compared with wild-type and heterozygotes, which are indistinguishable. (Hematoxylin and eosin stained; $\times 40$ ). 
$\beta 3^{+/-}$

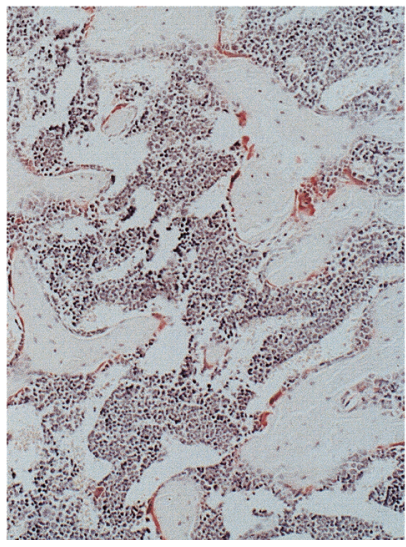

$\beta 3^{-1-}$

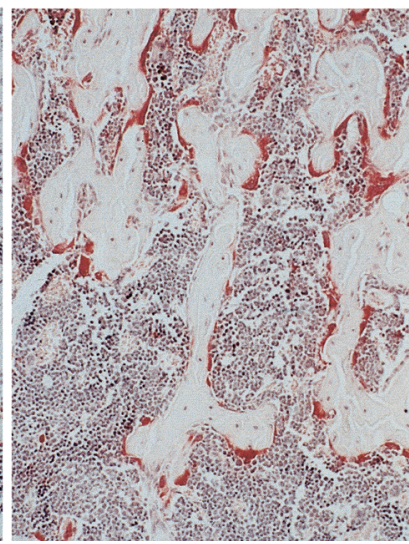

Figure 7

$\beta 3^{-/-}$mice generate increased numbers of osteoclasts. Histologic sections of distal femoral metaphysic of 2 -month-old $\beta 3^{+/-}$and $\beta 3^{-/-}$littermates were stained for TRAP activity (red reaction product) to identify osteoclasts that are increased in the mutant $(\times 250)$.

cally, the mean maximum depth of lacunae excavated by $\beta 3^{+/}$osteoclasts is $12.9 \mu \mathrm{m} \pm 2.9(\mathrm{SD})$, whereas that of pits generated by $\beta 3^{-/-}$osteoclasts is $8.0 \mu \mathrm{m} \pm$ $0.8(\mathrm{SD})(P<0.001)$. Thus, $\alpha \mathrm{v} \beta 3$ is not required to initiate osteoclastic bone resorption, but is essential for its optimal expression.

\section{Discussion}

The $\alpha v$ family of integrins are heterodimeric, transmembrane molecules that both anchor cells to and transmit signals from the extracellular matrix. In fact, $\beta$ integrin subunits contain cytoplasmic domains that, upon matrix recognition, serve as a nidus for the attachment of cytoskeletal proteins and signaling molecules, thereby dictating cell shape and motility (20).

Net bone degradation may reflect either the mean resorptive capacity of the mature polykaryon and/or the rates at which osteoclasts differentiate and die. Given this complex scenario, there are many possibilities by which $\alpha v \beta 3$ may regulate skeletal mass. We posited, for example, that the integrin heterodimer may merely serve as a matrix-anchoring molecule, or, alternatively, it may also transmit intracellular signals initiating the resorptive process (21). Evidence has subsequently appeared, however, confirming that osteoclast $\alpha v \beta 3$ transmits extracellular signals leading to phosphorylation of key integrin-associated signaling proteins such as pyk 2 (22). This information, although suggestive, does not prove that $\alpha v \beta 3$-mediated matrix-derived signals regulate the osteoclast's capacity to resorb bone.

To address these issues, we generated $\beta 3$ knockout mice that, as expected, exhibit Glanzmann's thrombasthenia (8). These mutants grow normally, and, by external examination, they are indistinguishable from their wild-type and heterozygous counterparts. Thus, lack of $\alpha v \beta 3$ does not impact skeletal model- ing or sculpting; this is an important consideration if inhibitors of the integrin are to be administered to osteopenic children.

Unlike previously reported in vitro (8) and in vivo (4) inhibitor studies implying $\alpha v \beta 3$ occupancy is necessary for optimal osteoclastogenesis, $\beta 3^{-/-}$mice not only contain osteoclasts but do so in substantially increased numbers. Given the hypocalcemia extant in the mutant mice, hyperparathyroidism likely prompts exuberant osteoclastogenesis. A similar scenario holds true in osteopetrosis (23).

$\beta 3$ osteoclasts are, however, morphologically and functionally abnormal. This dysfunction reflects failure to optimally organize the cytoskeleton upon matrix contact and is consistent with our suggestion that polarization of the cell's proton pump to its resorptive microenvironment reflects intracellular signals originating by recognition of $\alpha v \beta 3$ ligand (21). In this regard, failure to form normal ruffled membranes and the striking cytoskeletal abnormalities of $\alpha v \beta 3$-deficient osteoclasts suggest that the integrin functions not merely as an anchoring molecule, but also transmits essential, matrix-derived, intraosteoclastic signals. Proof of this posture will require distinction of the events mediated by only ligand recognition from intracellular signals transmitted by the integrin.

The impaired resorption extant in various osteopetrotic mutant mouse strains may represent failure to recruit osteoclasts (24) or diminished activity of the terminal polykaryon (25). The abundance of osteoclasts in the $\beta 3^{-/-}$mouse, also occurring in other states of resorptive insufficiency, both murine (25) and human (18), establishes that the resorptive
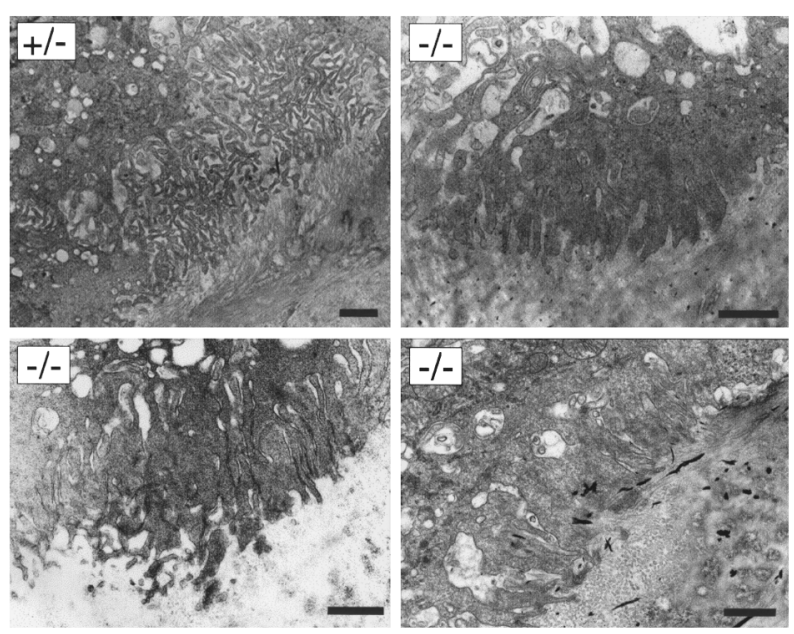

\section{Figure 8}

Ruffled membrane formation in osteoclasts of $\beta 3^{-/-}$mice is abnormal. Electron micrographs of osteoclasts resident in metaphyseal bone of $\beta 3^{+/-}$and $\beta 3^{-/-}$mice. The ruffled membranes of heterozygous osteoclasts are indistinguishable from wild-type, whereas those of each $\beta 3^{-/-}$osteoclast consists of thickened and blunted villous structures. Scale bar: $1.0 \mu \mathrm{m}$. 
$\beta 3^{+/-}$
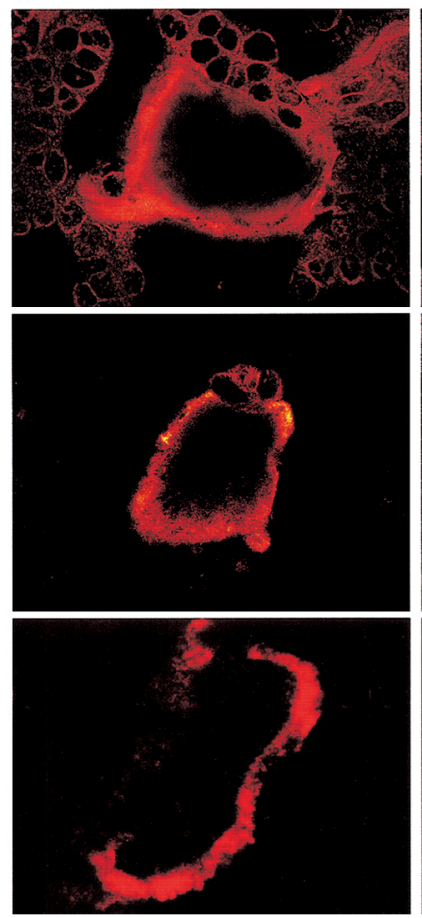

\section{Figure 9}

Actin organization in $\beta 3^{-/-}$osteoclasts is abnormal. $\beta 3^{+/-}$and $\beta 3^{-/-}$marrow was blotted onto slides. The cells were stained for TRAP activity, permeabilized, and then incubated with rhodamine-phalloidin. TRAP-expressing multinucleated cells were identified and $f$-actin was visualized by confocal microscopy. $\beta 3^{+/-}$ osteoclasts develop a characteristic actin ring, whereas F-actin in $\beta 3$ osteoclasts is diffusely distributed throughout the cytoplasm.

defect in this mutant arises from diminished bone degradation by the mature cell and not from arrested osteoclastogenesis. On the other hand, osteoclast recruitment, a process requiring cell-matrix attachment, is brisk in the absence of $\alpha v \beta 3$. Thus, an attachment molecule other than $\alpha v \beta 3$ must mediate matrix recognition by $\beta 3^{-/-}$osteoclast precursors sufficient to permit differentiation, but insufficient to sustain normal bone degradation.

Macrophages, upon isolation from marrow, contain abundant $\alpha v \beta 5$ and little $\alpha v \beta 3$ (17). With commitment to the osteoclast phenotype, the $\beta 5$ subunit is progressively replaced by $\beta 3$. This observation raised the possibility that upon $\beta 3$ deletion, $\alpha v \beta 5$ persists and is functionally redundant. Such is not the case as osteoclast differentiation of both wild-type and mutant BMMs is attended by loss of $\alpha v \beta 5$. We find, however, that $\beta 3^{-/-}$osteoclasts detach in the presence of collagenase and spread on native collagen (not shown), suggesting - but not proving - that collagen may serve as an attachment substrate sufficient for osteoclast differentiation, whereas optimal resorption requires $\alpha v \beta 3$.

In contrast with studies of the role of $\alpha v \beta 3$ in angiogenesis, wherein results with inhibitors (6) and genetic ablation $(7,8)$ are not in agreement, most of our obser-

vations conform with inhibitor experiments $(3,4)$. On the other hand, the potent $\alpha v \beta 3$ ligand, echistatin dampens osteoclast formation in vitro (5), and an RGD peptidomimetic decreases osteoclast number in vivo (4). Thus, the $\beta 3$ knockout mouse permits us to conclude that, in physiological circumstances, $\alpha v \beta 3$ is probably not essential for osteoclastogenesis.

Perhaps the most challenging aspect of the $\alpha v \beta 3$ knockout mouse is the relatively late appearance of profound osteosclerosis. The ability of $\beta 3^{-/-}$osteoclasts to initiate resorptive lacunae on dentin (albeit suboptimal), taken with normal skeletal development, tooth eruption, and resorption of physeal cartilage suggests bone remodeling proceeds in the absence of $\alpha v \beta 3$. The fact that osteoclasts derived from 4- to 8-week-old mutants are dysfunctional indicates that the resorptive defect is not one acquired with age, but is instead the cumulative manifestation of partial osteoclast arrest. Thus, a reasonable hypothesis holds that although osteoclasts are dysfunctional in $\alpha v \beta 3$-deficient mice, deployment of a larger than normal number of these cells initially achieves a reasonable net resorptive rate. With time, however, $\beta 3$-null osteoclasts eventually fail to keep pace with bone deposition and osteosclerosis ensues.

Maintenance of skeletal integrity requires its continued remodeling (26). Hence, complete arrest of bone resorption is an undesirable feature of a potential bone-sparing agent. The fact, therefore, that bone resorption is not completely arrested in $\beta 3$-null animals buttresses $\alpha v \beta 3$ inhibition as a potential antiosteoporosis strategy.

$\beta 3^{+1}$
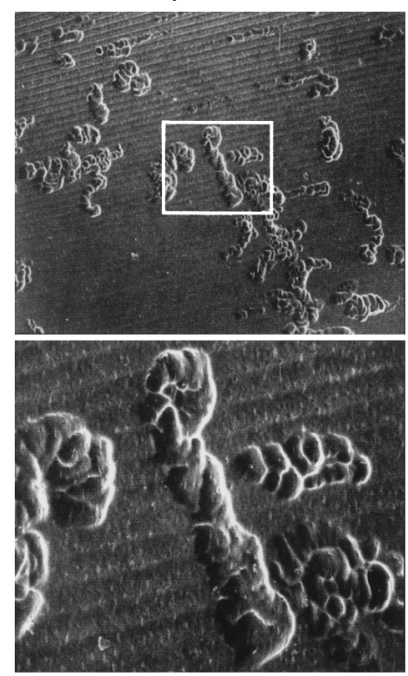

\section{Figure 10}

The resorptive capacity of $\beta 3^{-/-}$osteoclasts is defective. Osteoclasts were generated by culturing $\beta 3^{+/-}$or $\beta 3^{-/-}$BMMs with ST2 cells on dentin. After 7 days, the cells were removed, and resorption lacuna visualized by scanning electron microscopy. Whereas $\beta 3^{+/-}$osteoclasts produce numerous, well defined resorption lacunae, those formed by $\beta 3^{-/-}$osteoclasts have indistinct borders and are shallow. 


\section{Acknowledgments}

We thank G. Papodemitirou of the University of Western Australia for his help. This work was supported by grants from the National Institutes of Health (NIH) (AR42404 to F.P. Ross; DE05413, AR46523, and AR32788 to S.L. Teitelbaum; National Heart, Lung, and Blood Institute grant HL41484 to R.O. Hynes; NIH grant GM07200 to J. Lam; NIH grant AR08335 to K.P. McHugh); Shriners' Hospital for Crippled Children (grant 8560 to S.L. Teitelbaum); Alpha Omega Alpha Medical Honor Society 1998 Student Research Fellowship (to J. Lam); and by the Howard Hughes Medical Institute. K. Hodivala-Dilke was a fellow of the Human Frontiers Science Program and the Dystrophic Epidermolysis Bullosa Research Association. R.O. Hynes is an investigator of the Howard Hughes Medical Institute.

1. Lacey, D.L., et al. 1998. Osteoprotegerin ligand is a cytokine that regulates osteoclast differentiation and activation. Cell. 93:165-176.

2. Blair, H.C., Teitelbaum, S.L., Ghiselli, R., and Gluck, S. 1989. Osteoclastic bone resorption by a polarized vacuolar proton pump. Science. 245:855-857.

3. Horton, M.A., Taylor, M.L., Arnett, T.R., and Helfrich, M.H. 1991. Arggly-asp (RGD) peptides and the anti-vitronectin receptor antibody $23 \mathrm{C6}$ inhibit dentine resorption and cell spreading by osteoclasts. Exp. Cell Res. 195:368-375.

4. Engleman, V.W., et al. 1997. A peptidomimetic antagonist of the $\alpha v \beta 3$ integrin inhibits bone resorption in vitro and prevents osteoporosis in vivo. J. Clin. Invest. 99:2284-2292.

5. Nakamura, I., Tanaka, H., Rodan, G.A., and Duong, L.T. 1998. Echistatin inhibits the migration of murine prefusion osteoclasts and the formation of multinucleated osteoclast-like cells. Endocrinology. 139:5182-5193.

6. Storgard, C.M., et al. 1999. Decreased angiogenesis and arthritic disease in rabbits treated with an $\alpha \mathrm{v} \beta 3$ antagonist. J. Clin. Invest. 103:47-54.

7. Bader, B.L., Rayburn, H., Crowley, D., and Hynes, R.O. 1998. Extensive vasculogenesis, angiogenesis, and organogenesis precede lethality in mice lacking all alpha v integrins. Cell. 95:507-519.

8. Hodivala-Dilke, K.M., et al. 1999. $\beta 3$ integrin-deficient mice are a model for Glanzmann thrombasthenia showing placental defects and reduced survival. J. Clin. Invest. 103:229-238.

9. Diaz-Gonzalez, F., Forsyth, J., Steiner, B., and Ginsberg, M.H. 1996 Trans-dominant inhibition of integrin function. Mol. Biol. Cell. 7:1939-1951.

10. Kitazawa, S., Ross, F.P., McHugh, K., and Teitelbaum, S.L. 1995. Inter- leukin- 4 induces expression of the integrin $\alpha v \beta 3$ via transactivation of the $\beta 3$ gene. J. Biol. Chem. 270:4115-4120.

11. Abu-Amer, Y., et al. 1998. Tumor necrosis factor-alpha activation of nuclear transcription factor- $\kappa \mathrm{B}$ in marrow macrophages is mediated by c-Src tyrosine phosphorylation of Iк B $\alpha$. J. Biol. Chem. 273:29417-29423.

12. Abu-Amer, Y., Ross, F.P., Edwards, J., and Teitelbaum, S.L. 1997. Lipopolysaccharide-stimulated osteoclastogenesis is mediated by tumor necrosis factor via its p55 receptor. J. Clin. Invest. 100:1557-1565.

13. Clohisy, D.R., Bar-Shavit, Z., Chappel, J., and Teitelbaum, S.L. 1987. 1,25Dihydroxyvitamin D3 modulates bone marrow macrophage precursor proliferation and differentiation: upregulation of the mannose receptor. J. Biol. Chem. 262:15922-15929.

14. McHugh, K.P., Teitelbaum, S.L., Kitazawa, S., and Ross, F.P. 2000 Cloning and characterization of the murine $\beta 3$ integrin gene promoter: identification of an interleukin- 4 responsive element and its regulation by Stat-6. J. Bone Miner. Res. In press.

15. Feng, X., Teitelbaum, S.L., Quiroz, M., Towler, D.A., and Ross, F.P. 1999. Cloning of the murine $\beta 5$ integrin subunit promoter. Identification of a novel sequence mediating granulocyte-macrophage colony-stimulating factor-dependent repression of $\beta 5$ integrin gene transcription. J. Biol. Chem. 274:1366-1374.

16. Greenfield, E.M., et al. 1992. Avian osteoblast conditioned media stimulate bone resorption by targeting multinucleating osteoclast precursors. Calcif. Tissue Int. 51:317-323.

17. Inoue, M., Namba, N., Chappel, J., Teitelbaum, S.L., and Ross, F.P. 1998. Granulocyte macrophage colony stimulating factor reciprocally regulates $\alpha_{v}$-associated integrins on murine osteoclast precursors. Mol. Endocrinol. 12:1955-1962.

18. Coccia, P.F., et al. 1980. Successful bone-marrow transplantation for infantile malignant osteopetrosis. N. Engl. J. Med. 302:701-708.

19. Holtrop, M.E. 1991. Light and electron microscopic structure of osteoclasts. In Bone. B.K. Hall, editor. CRC Press. Boca Raton, FL. 1-29.

20. Schwartz, M.A., Schaller, M.D., and Ginsberg, M.H. 1995. Integrins: emerging paradigms of signal transduction. Annu. Rev. Cell Dev. Biol. 11:549-599.

21. Abu-Amer, Y., Ross, F.P., Schlesinger, P., Tondravi, M.M., and Teitelbaum, S.L. 1997. Substrate recognition by osteoclast precursors induces s-crc/microtubule association. J. Cell Biol. 137:247-258.

22. Duong, L.T., et al. 1998. PYK2 in osteoclasts is an adhesion kinase, localized in the sealing zone, activated by ligation of $\alpha(\mathrm{v}) \beta 3$ integrin, and phosphorylated by src kinase. J. Clin. Invest. 102:881-892.

23. Cournot, G., et al. 1992. Mineral metabolism in infants with malignant osteopetrosis: heterogeneity in plasma 1,25-dihydroxyvitamin D levels and bone histology. J. Bone Miner. Res. 7:1-10.

24. Tondravi, M.M., et al. 1997. Targeted deletion of PU.1 induces osteopetrosis rescuable by bone marrow transplantation. Nature. 386:81-84.

25. Soriano, P., Montgomery, C., Geske, R., and Bradley, A. 1991. Targeted disruption of the c-src proto-oncogene leads to osteopetrosis in mice. Cell. 64:693-702.

26. Delmez, J.A., Dusso, A.S., Slatopolsky, E., and Teitelbaum, S.L. 1995 Modulation of renal osteodystrophy by extrarenal production of calcitriol. Am. J. Nephrol. 15:85-89. 